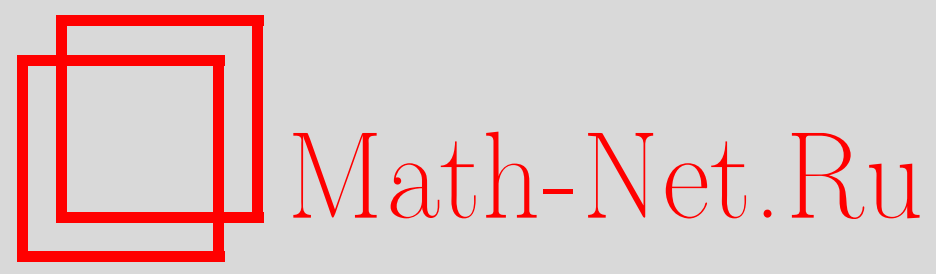

T. K. Юлдашев, Обратная задача для обыкновенного интегро-дифференциального уравнения с вырожденным ядром и нелокальными интегральными условиями, Вестник ТвГУ. Серия: Прикладная математика, 2016, выпуск 3, 19-33

DOI: https://doi.org/10.26456/vtpmk18

Использование Общероссийского математического портала Math-Net.Ru подразумевает, что вы прочитали и согласны с пользовательским соглашением

http://www.mathnet.ru/rus/agreement

Параметры загрузки:

IP : 3.85 .7 .115

26 апреля 2023 г., 10:02:40 
УДК 517.9

\section{ОБРАТНАЯ ЗАДАЧА ДЛЯ ОБЫКНОВЕННОГО ИНТЕГРО-ДИФФЕРЕНЦИАЛЬНОГО УРАВНЕНИЯ С ВЫРОЖДЕННЫМ ЯДРОМ И НЕЛОКАЛЬНЫМИ ИНТЕГРАЛЬНЫМИ УСЛОВИЯМИ}

\section{Юлдашев Т.K.}

Сибирский государственный аэрокосмический университет имени академика М.Ф. Решетнева, г. Красноярск

Поступила в редакцию 10.09.2016, после переработки 30.09.2016.

Рассмотрены вопросы однозначной разрешимости нелокальной обратной задачи по определению источника и граничного режима для нелинейного обыкновенного интегро-дифференциального уравнения Фредгольма второго порядка с вырожденным ядром. С принятием обозначения интегро-дифференциальное уравнение сведено к системе алгебраических уравнений с нелинейной правой частью. Эта система решена модифицированным методом Крамера. С помощью дополнительных условий получена система из двух уравнений относительно первых двух неизвестных величин. Доказана однозначная разрешимость этой системы методом последовательньх приближений. Выведена формула для определения третьей неизвестной величины. Изучена непрерывная зависимость решения уравнения от величин восстановления.

Ключевые слова: нелокальная обратная задача, интегродифференциальное уравнение, вырожденное ядро, система алгебраических уравнений, однозначная разрешимость.

Вестник ТвГУ. Серия: Прикладная математика. 2016. № 3. С. 19-33.

\section{Введение}

Математическое моделирование многих процессов, происходящих в реальном мире, часто приводит к изучению начальных и граничньх задач для обыкновенных дифференциальных уравнений и дифференциальных уравнений в частных производных. Такие задачи составляют основу математической физики. Представляют большой интерес с точки зрения физических приложений интегро-дифференциальные уравнения. Изучению обыкновенных интегродифференциальных уравнений посвящено большое количество работ (см., напр. $[1-8])$.

Часто на практике встречаются ситуации, когда объект исследования либо недоступен для наблюдения, либо проведение такого эксперимента дорого. Процедура решения таких задач связана с преодолением серьезных математических 
трудностей. Успех ее сильно зависит как от качества и количества полученной из эксперимента информации, так и от способа ее обработки.

Теория обратных задач представляет собой активно развивающееся направление современной теории дифференциальных уравнений. К обратным задачам относят задачи определения некоторых физических свойств объектов, таких, как плотность, коэффициент теплопроводности, упругие модули в зависимости от координат или в виде функций других параметров. Заметим, что без умения решать прямые задачи невозможно подойти к обратным. Линейные обратные задачи рассматривались во многих работах, в частности, в [9-18]. В настоящее время совершенствуется и методика решений обратных задач.

В случаях решения задач математического моделирования, когда граница области протекания физического процесса недоступна для измерений, в качестве дополнительной информации, достаточной для однозначной разрешимости задачи, могут служить нелокальные условия в интегральной форме [19-21]. Метод вырожденного ядра для интегро-дифференциальных уравнений в частных производных рассматривались в работах [22-24].

\section{1. Постановка задачи}

В настоящей работе предлагается методика изучения обратной краевой задачи для обыкновенного интегро-дифференциального уравнения Фредгольма второго порядка с вырожденным ядром.

На отрезке $\Omega \equiv[0 ; T]$ рассматривается уравнение вида

$$
u^{\prime \prime}(t)+\mu \int_{0}^{T} K(t, s) u(s) d s=\eta(t) \beta+f\left(\beta, \int_{0}^{T} H(\theta) u(\theta) d \theta\right)
$$

с нелокальными интегральными

$$
u(0)+\int_{0}^{T} \Theta_{1}(t) u(t) d t=\varphi_{1}, \quad u^{\prime}(0)+\int_{0}^{T} \Theta_{2}(t) u(t) d t=\varphi_{2}
$$

и дополнительными условиями

$$
\int_{0}^{T} \Theta_{3}(t) u(t) d t=\psi, \quad u^{\prime}(T)=\nu
$$

где $u(t) \in C^{2}(\Omega)$ - основная неизвестная функция, $\eta(t) \in C(\Omega), f(\beta, \gamma) \in C(R \times R)$, $\beta \in R$ - первая величина восстановления, $\psi, \nu \equiv$ const, $K(t, s)=\sum_{i=1}^{m} a_{i}(t) b_{i}(s)$, $a_{i}(t), b_{i}(s) \in C(\Omega), \varphi_{k} \equiv$ const $, k=1,2, \varphi_{2}$ - вторая величина восстановления, $\int_{0}^{T}|H(t)| d t<\infty, \Theta_{k}(t) \in C^{2}(\Omega), k=\overline{1,3}, 0<T<\infty, \mu$ - спектральный параметр. Здесь предполагается, что функции $a_{i}(t)$ и $b_{i}(s)$ являются линейно независимыми. 
Определение 1. Под решением обратной задачи задачи (1)-(3) понимаем тройку величин $\left\{u(t) \in C^{2}(\Omega), \beta \in R, \varphi_{2} \in R\right\}$, удовлетворяющих уравнению (1) $u$ условиям (2), (3).

\section{2. Вывод формул для вычисления неизвестных величин}

Уравнение (1) запишем в следующем виде

$$
u^{\prime \prime}(t)+\mu \int_{0}^{T} \sum_{i=1}^{m} a_{i}(t) b_{i}(s) u(s) d s=\eta(t) \beta+f(\cdot),
$$

где $f(\cdot)=f\left(\beta, \int_{0}^{T} H(\theta) u(\theta) d \theta\right)$.

$\mathrm{C}$ помощью обозначения

$$
c_{i}=\int_{0}^{T} b_{i}(s) u(s) d s
$$

уравнение (4) перепишется так

$$
u^{\prime \prime}(t)=-\mu \sum_{i=1}^{m} a_{i}(t) c_{i}+\eta(t) \beta+f(\cdot) .
$$

Правую часть (6) обозначим через $G(t)$. Тогда путем интегрирования два раза по $t$ из (6) получаем

$$
u(t)=C_{2}+C_{1} t+\int_{0}^{t}(t-s) G(s) d s
$$

где $C_{1}, C_{2}$ - пока неизвестные постоянные, для определения которых из интегральных условий (2) получаем следующую систему алгебраических уравнений

$$
\left\{\begin{array}{l}
C_{1} \alpha_{3}+C_{2} \alpha_{1}=g_{1}, \\
C_{1} \alpha_{2}+C_{2} \alpha_{4}=g_{2},
\end{array}\right.
$$

где $\alpha_{1}=1+\int_{0}^{T} \Theta_{1}(t) d t, \alpha_{2}=1+\int_{0}^{T} \Theta_{2}(t) t d t, \alpha_{3}=\int_{0}^{T} \Theta_{1}(t) t d t, \alpha_{4}=\int_{0}^{T} \Theta_{2}(t) d t$, $g_{k}=\varphi_{k}-\int_{0}^{T} \Theta_{k}(t) \int_{0}^{t}(t-s) G(s) d s d t, k=1,2$.

Пусть

$$
\omega=\alpha_{3} \alpha_{4}-\alpha_{1} \alpha_{2} \neq 0 .
$$

Тогда, решая систему алгебраических уравнений $(8)$, из (7) получаем

$$
u(t)=\frac{1}{\omega}\left[\left(\alpha_{2}+\alpha_{3} t\right) \varphi_{1}-\left(\alpha_{1} t+\alpha_{3}\right) \varphi_{2}\right]+
$$




$$
\begin{aligned}
& +\mu \frac{\alpha_{2}+\alpha_{3} t}{\omega} \int_{0}^{T} \Theta_{1}(t) \int_{0}^{t}(t-s) \sum_{i=1}^{m} q_{i}(s) c_{i} d s d t- \\
& -\mu \frac{\alpha_{3}+\alpha_{1} t}{\omega} \int_{0}^{T} \Theta_{2}(t) \int_{0}^{t}(t-s) \sum_{i=1}^{m} q_{i}(s) c_{i} d s d t- \\
& -\mu \int_{0}^{t}(t-s) \sum_{i=1}^{m} q_{i}(s) c_{i} d s+\beta W_{1}(t)+f(\cdot) W_{2}(t),
\end{aligned}
$$

где

$$
\begin{gathered}
W_{1}(t)=\int_{0}^{t}(t-s) p(s) d s-\frac{\alpha_{2}+\alpha_{3} t}{\omega} \int_{0}^{T} \Theta_{1}(t) \int_{0}^{t}(t-s) p(s) d s d t+ \\
\quad+\frac{\alpha_{3}+\alpha_{1} t}{\omega} \int_{0}^{T} \Theta_{2}(t) \int_{0}^{t}(t-s) p(s) d s d t \\
W_{2}(t)=\int_{0}^{t}(t-s) s^{2} d s-\frac{\alpha_{2}+\alpha_{3} t}{\omega} \int_{0}^{T} \Theta_{1}(t) \int_{0}^{t}(t-s) s^{2} d s d t+ \\
\quad+\frac{\alpha_{3}+\alpha_{1} t}{\omega} \int_{0}^{T} \Theta_{2}(t) \int_{0}^{t}(t-s) s^{2} d s d t, \\
q_{i}(t)=\int_{0}^{t}(t-s) a_{i}(s) d s, \quad p(t)=\int_{0}^{t}(t-s) \eta(s) d s, i=\overline{1, m} .
\end{gathered}
$$
(CAY)

Подстановка выражения (10) в (5) дает систему алгебраических уравнений

$$
c_{i}+\mu \sum_{j=1}^{m} A_{i j} c_{j}=B_{i}, i=\overline{1, m}
$$

где

$$
\begin{gathered}
A_{i j}=\int_{0}^{T} b_{i}(s) \frac{\alpha_{2}+\alpha_{3} s}{\omega} \int_{0}^{T} \Theta_{1}(\zeta) \int_{0}^{\zeta}(\zeta-\xi) q_{j}(\xi) d \xi d \zeta d s- \\
-\int_{0}^{T} b_{i}(s) \frac{\alpha_{3}+\alpha_{1} s}{\omega} \int_{0}^{T} \Theta_{2}(\zeta) \int_{0}^{\zeta}(\zeta-\xi) q_{j}(\xi) d \xi d \zeta d s-\int_{0}^{T} b_{i}(s) \int_{0}^{s}(s-\zeta) q_{j}(\zeta) d \zeta d s
\end{gathered}
$$

и

$$
B_{i}=\frac{1}{\omega} \int_{0}^{T} b_{i}(s)\left[\left(\alpha_{2}+\alpha_{3} s\right) \varphi_{1}-\left(\alpha_{1} s+\alpha_{3}\right) \varphi_{2}\right] d s-
$$




$$
-\int_{0}^{T} b_{i}(s)\left[\beta W_{1}(s)+f(\cdot) W_{2}(s)\right] d s .
$$

САУ (11) однозначно разрешима при любых конечных $B_{i}$, если выполняется следующее условие

$$
\Delta(\mu)=\left|\begin{array}{cccc}
1+\mu A_{11} & \mu A_{12} & \cdots & \mu A_{1 m} \\
\mu A_{21} & 1+\mu A_{22} & \cdots & \mu A_{2 m} \\
\vdots & \vdots & \ddots & \vdots \\
\mu A_{m 1} & \mu A_{m 2} & \cdots & 1+\mu A_{m m}
\end{array}\right| \neq 0 .
$$

Определитель $\Delta(\mu)$ в (13) есть многочлен относительно $\mu$ степени не выше $m$. Уравнение $\Delta(\mu)=0$ имеет не более $m$ различных корней. Эти корни являются собственными числами ядра интегро-дифференциального уравнения (1). Другие значения $\mu$ называются регулярными, при которых условие (13) выполняется. Для регулярных значений $\mu$ система (11) имеет единственное решение при ненулевой конечной правой части. В настоящей работе для таких регулярных значений параметра $\mu$ устанавливается однозначная разрешимость поставленной обратной задачи (1)-(3).

Решения САУ (11) записываем в виде

$$
c_{i}=\frac{\Delta_{i}(\mu)}{\Delta(\mu)}, i=\overline{1, m},
$$

где $\Delta_{i}(\mu)=$

$$
=\left|\begin{array}{ccccccc}
1+\mu A_{11} & \ldots & \mu A_{1(i-1)} & B_{1} & \mu A_{1(i+1)} & \ldots & \mu A_{1 m} \\
\mu A_{21} & \ldots & \mu A_{2(i-1)} & B_{2} & \mu A_{2(i+1)} & \ldots & \mu A_{2 m} \\
\vdots & \vdots & \vdots & \vdots & \vdots & \ddots & \vdots \\
\mu A_{m 1} & \ldots & \mu A_{m(i-1)} & B_{m} & \mu A_{m(i+1)} & \ldots & 1+\mu A_{m m}
\end{array}\right| .
$$

Среди элементов определителей $\Delta_{i}(\mu)$ находятся $B_{i}$. В свою очередь, в составе $B_{i}$ находятся неизвестные величины $u(t), \beta$ и $\varphi_{2}$. В самом деле, эти неизвестные величины находились в правой части САУ (11). Для вывода их из знака определителя выражение в (12) запишем в следующем виде

$$
B_{i}=B_{1 i}+B_{2 i} \varphi_{2}+B_{3 i} \beta+B_{4 i} f(\cdot),
$$

где

$$
\begin{gathered}
B_{1 i}=\frac{\varphi_{1}}{\omega} \int_{0}^{T} b_{i}(s)\left(\alpha_{2}+\alpha_{3} s\right) d s, \quad B_{2 i}=-\frac{1}{\omega} \int_{0}^{T} b_{i}(s)\left(\alpha_{3}+\alpha_{1} s\right) d s, \\
B_{3 i}=\int_{0}^{T} b_{i}(s) W_{1}(s) d s, \quad B_{4 i}=\int_{0}^{T} b_{i}(s) W_{2}(s) d s .
\end{gathered}
$$

В этом случае, согласно свойству определителя имеем

$$
\Delta_{i}(\mu)=\Delta_{1 i}(\mu)+\varphi_{2} \Delta_{2 i}(\mu)+\beta \Delta_{3 i}(\mu)+f(\cdot) \Delta_{4 i}(\mu),
$$


где

$\Delta_{k i}(\mu)=\left|\begin{array}{ccccccc}1+\mu A_{11} & \ldots & \mu A_{1(i-1)} & B_{k 1} & \mu A_{1(i+1)} & \ldots & \mu A_{1 m} \\ \mu A_{21} & \ldots & \mu A_{2(i-1)} & B_{k 2} & \mu A_{2(i+1)} & \ldots & \mu A_{2 m} \\ \vdots & \vdots & \vdots & \vdots & \vdots & \ddots & \vdots \\ \mu A_{m 1} & \ldots & \mu A_{m(i-1)} & B_{k m} & \mu A_{m(i+1)} & \ldots & 1+\mu A_{m m}\end{array}\right|, k=\overline{1,4}$.

Тогда формула (14) переписывается в виде

$$
c_{i}=\frac{\Delta_{1 i}(\mu)}{\Delta(\mu)}+\varphi_{2} \frac{\Delta_{2 i}(\mu)}{\Delta(\mu)}+\beta \frac{\Delta_{3 i}(\mu)}{\Delta(\mu)}+f(\cdot) \frac{\Delta_{4 i}(\mu)}{\Delta(\mu)}, i=\overline{1, m} .
$$

Подставляя (15) в (10), имеем следующее интегральное уравнение

$$
u(t)=Q_{1}(t)+Q_{2}(t) \varphi_{2}+Q_{3}(t) \beta+Q_{4}(t) f(\cdot),
$$

где

$$
\begin{aligned}
& Q_{1}(t)=\frac{1}{\omega}\left(\alpha_{2}+\alpha_{3} t\right) \varphi_{1}-\mu \int_{0}^{t}(t-s) \sum_{i=1}^{m} q_{i}(s) \frac{\Delta_{1 i}(\mu)}{\Delta(\mu)} d s+ \\
& +\mu \frac{\alpha_{2}+\alpha_{3} t}{\omega} \int_{0}^{T} \Theta_{1}(t) \int_{0}^{t}(t-s) \sum_{i=1}^{m} q_{i}(s) \frac{\Delta_{1 i}(\mu)}{\Delta(\mu)} d s d t- \\
& -\mu \frac{\alpha_{3}+\alpha_{1} t}{\omega} \int_{0}^{T} \Theta_{2}(t) \int_{0}^{t}(t-s) \sum_{i=1}^{m} q_{i}(s) \frac{\Delta_{1 i}(\mu)}{\Delta(\mu)} d s d t, \\
& Q_{2}(t)=-\frac{\alpha_{3}+\alpha_{1} t}{\omega}-\mu \int_{0}^{t}(t-s) \sum_{i=1}^{m} q_{i}(s) \frac{\Delta_{2 i}(\mu)}{\Delta(\mu)} d s+ \\
& +\mu \frac{\alpha_{2}+\alpha_{3} t}{\omega} \int_{0}^{T} \Theta_{1}(t) \int_{0}^{t}(t-s) \sum_{i=1}^{m} q_{i}(s) \frac{\Delta_{2 i}(\mu)}{\Delta(\mu)} d s d t- \\
& -\mu \frac{\alpha_{3}+\alpha_{1} t}{\omega} \int_{0}^{T} \Theta_{2}(t) \int_{0}^{t}(t-s) \sum_{i=1}^{m} q_{i}(s) \frac{\Delta_{2 i}(\mu)}{\Delta(\mu)} d s d t, \\
& Q_{3}(t)=\mu \tau_{n} \frac{\alpha_{2}+\alpha_{3} t}{\omega} \int_{0}^{T} \Theta_{1}(t) \int_{0}^{t}(t-s) \sum_{i=1}^{m} q_{i}(s) \frac{\Delta_{3 i}(\mu)}{\Delta(\mu)} d s d t- \\
& -\mu \frac{\alpha_{3}+\alpha_{1} t}{\omega} \int_{0}^{T} \Theta_{2}(t) \int_{0}^{t}(t-s) \sum_{i=1}^{m} q_{i}(s) \frac{\Delta_{3 i}(\mu)}{\Delta(\mu)} d s d t- \\
& -\mu \int_{0}^{t}(t-s) \sum_{i=1}^{m} q_{i}(s) \frac{\Delta_{3 i}(\mu)}{\Delta(\mu)} d s+W_{1}(t),
\end{aligned}
$$




$$
\begin{gathered}
Q_{4}(t)=\mu \frac{\alpha_{2}+\alpha_{3} t}{\omega} \int_{0}^{T} \Theta_{1}(t) \int_{0}^{t}(t-s) \sum_{i=1}^{m} q_{i}(s) \frac{\Delta_{4 i}(\mu)}{\Delta(\mu)} d s d t- \\
-\mu \frac{\alpha_{3}+\alpha_{1} t}{\omega} \int_{0}^{T} \Theta_{2}(t) \int_{0}^{t}(t-s) \sum_{i=1}^{m} q_{i}(s) \frac{\Delta_{4 i}(\mu)}{\Delta(\mu)} d s d t- \\
-\mu \int_{0}^{t}(t-s) \sum_{i=1}^{m} q_{i}(s) \frac{\Delta_{4 i}(\mu)}{\Delta(\mu)} d s+W_{2}(t) .
\end{gathered}
$$

Для определения первой величины восстановления воспользуемся первым условием из (2):

$$
\begin{aligned}
\psi=\int_{0}^{T} \Theta_{3}(t) Q_{1}(t) d t & +\varphi_{2} \int_{0}^{T} \Theta_{3}(t) Q_{2}(t) d t+\beta \int_{0}^{T} \Theta_{3}(t) Q_{3}(t) d t+ \\
& +f(\cdot) \int_{0}^{T} \Theta_{3}(t) Q_{4}(t) d t .
\end{aligned}
$$

Пусть $\int_{0}^{T} \Theta_{3}(t) Q_{3}(t) d t \neq 0$. Тогда из последнего соотношения имеем

$$
\beta=\gamma_{1}-\gamma_{2} \varphi_{2}-\gamma_{3} f(\cdot)
$$

где

$$
\gamma_{1}=\frac{\psi-\int_{0}^{T} \Theta_{3}(t) Q_{1}(t) d t}{\int_{0}^{T} \Theta_{3}(t) Q_{3}(t) d t}, \gamma_{2}=\frac{\int_{0}^{T} \Theta_{3}(t) Q_{2}(t) d t}{\int_{0}^{T} \Theta_{3}(t) Q_{3}(t) d t}, \gamma_{3}=\frac{\int_{0}^{T} \Theta_{3}(t) Q_{4}(t) d t}{\int_{0}^{T} \Theta_{3}(t) Q_{3}(t) d t} .
$$

Подставляя (17) в (16), получаем

$$
u(t)=F(t)+\varphi_{2} D(t)+E(t) f(\cdot),
$$

где $F(t)=Q_{1}(t)+\gamma_{1} Q_{3}(t), D(t)=Q_{2}(t)-\gamma_{2} Q_{3}(t), E(t)=Q_{4}(t)-\gamma_{3} Q_{3}(t)$.

Теперь воспользуемся вторым условием из (3). Тогда из (18) имеем

$$
\nu=F^{\prime}(T)+\varphi_{2} D^{\prime}(T)+E^{\prime}(T) f(\cdot) .
$$

Отсюда находим формулу для определения второй величины восстановления

$$
\varphi_{2}=\chi_{1}-\chi_{2} f\left(\beta, \int_{0}^{T} H(\theta) u(\theta) d \theta\right)
$$


где $\chi_{1}=\frac{\nu-F^{\prime}(T)}{D^{\prime}(T)}, \chi_{2}=\frac{E^{\prime}(T)}{D^{\prime}(T)}$.

Подставляя (19) в (18), для основной неизвестной функции получаем уравнение

$$
u(t)=\Im_{1}(\beta, u) \equiv g_{1}(t)+g_{2}(t) f\left(\beta, \int_{0}^{T} H(\theta) u(\theta) d \theta\right),
$$

где $g_{1}(t)=F(t)+\chi_{1} D(t), g_{2}(t)=E(t)-\chi_{2} D(t)$.

Теперь, подставляя (19) в (17), для первой величины восстановления получаем уравнение

$$
\beta=\Im_{2}(\beta, u)=\sigma_{1}-\sigma_{2} f\left(\beta, \int_{0}^{T} H(\theta) u(\theta) d \theta\right),
$$

где $\sigma_{1}=\gamma_{1}-\gamma_{2} \chi_{1}, \sigma_{2}=\gamma_{2} \chi_{2}-\gamma_{3}$.

Уравнения (20) и (21) образуют систему уравнений (СУ)

$$
\left\{\begin{array}{c}
u(t)=\Im_{1}(\beta, u) \\
\beta=\Im_{2}(\beta, u)
\end{array}\right.
$$

\section{3. Однозначная разрешимость обратной задачи (1)-(3)}

Изучим вопросы разрешимости СУ (22). С этой целью в пространстве непрерывных функций $C(\Omega)$ рассматривается норма

$$
\|u(t)\|=\max _{t \in \Omega}|u(t)|
$$

Теорема 1. Пусть выполняются условия (9) и (13). Если

1) $D^{\prime}(T) \neq 0 ; \int_{0}^{T} \Theta_{3}(t) Q_{3}(t) d t \neq 0, t \in \Omega$;

2) $\omega_{k}=\left\|g_{k}(t)\right\|<\infty ; \varepsilon_{k}=\left|\sigma_{k}\right|<\infty, k=1,2$;

3) $M_{0}=|f(0,0)|<\infty ; M_{1}=\int_{0}^{T}|H(t)| d t<\infty$;

4) $\left\|f\left(\beta_{1}, \gamma_{1}\right)-f\left(\beta_{2}, \gamma_{2}\right)\right\| \leq w_{1}\left|\beta_{1}-\beta_{2}\right|+w_{2}\left|\gamma_{1}-\gamma_{2}\right|, 0<w_{k}, k=1,2$;

5) $\rho=w\left(\varepsilon_{2}+\omega_{2}\right)<1$, где $w=\max \left\{w_{1} ; M_{1} w_{2}\right\}$,

то СУ (22) имеет единственную пару решений. Эту пару решений можно найти методом последовательных приближений:

$$
\left\{\begin{aligned}
\beta^{0} & =0, \beta^{j+1}=\Im_{2}\left(\beta^{j}, u^{j}\right) \\
u^{0}(t) & =0, u^{j+1}(t)=\Im_{1}\left(\beta^{j}, u^{j}\right) .
\end{aligned}\right.
$$

Кроме того, однозначно определяется вторая величина восстановления $\varphi_{2}$.

Доказательство. С учетом условий теоремы из приближения (23) получаем оценки

$$
\left|\beta^{1}-\beta^{0}\right| \leq\left|\sigma_{1}\right|+\left|\sigma_{2}\right||f(0,0)| \leq \varepsilon_{1}+\varepsilon_{2} M_{0}
$$




$$
\left\|u^{1}(t)-u^{0}(t)\right\| \leq\left\|g_{1}(t)\right\|+\left\|g_{2}(t)\right\||f(0,0)| \leq \omega_{1}+\omega_{2} M_{0} .
$$

Для произвольных разностей справедливы следующие оценки

$$
\begin{gathered}
\left|\beta^{j+1}-\beta^{j}\right| \leq\left|\sigma_{2}\right|\left[w_{1}\left|\beta^{j}-\beta^{j-1}\right|+w_{2} \int_{0}^{T}|H(\theta)|\left|u^{j}(\theta)-u^{j-1}(\theta)\right|\right] \leq \\
\leq \varepsilon_{2}\left[w_{1}\left|\beta^{j}-\beta^{j-1}\right|+w_{2} M_{1}\left\|u^{j}(t)-u^{j-1}(t)\right\|\right] \leq \\
\leq w \varepsilon_{2}\left[\left|\beta^{j}-\beta^{j-1}\right|+\left\|u^{j}(t)-u^{j-1}(t)\right\|\right] \\
\left\|u^{j+1}(t)-u^{j}(t)\right\| \leq w \omega_{2}\left[\left|\beta^{j}-\beta^{j-1}\right|+\left\|u^{j}(t)-u^{j-1}(t)\right\|\right] .
\end{gathered}
$$

Из (26) и (27) получаем

$$
\begin{gathered}
\left|\beta^{j+1}-\beta^{j}\right|+\left\|u^{j+1}(t)-u^{j}(t)\right\| \leq \\
\leq \rho\left(\left|\beta^{j}-\beta^{j-1}\right|+\left\|u^{j}(t)-u^{j-1}(t)\right\|\right) .
\end{gathered}
$$

Из оценки (24) следует, что оператор $\Im_{2}(\beta, u)$ в правой части (21) переводит шар $S_{1}\left(\beta, r_{1}\right)$ с радиусом $r_{1}=\varepsilon_{1}+\varepsilon_{2} M_{0}$ в себя. Из оценки (25) аналогично следует, что оператор $\Im_{1}(\beta, u)$ в правой части (20) переводит шар $S_{2}\left(u, r_{2}\right)$ с радиусом $r_{2}=\omega_{1}+\omega_{2} M_{0}$ в себя. В силу последнего условия теоремы, из оценки (28) следует, что операторы в правой части (22) являются сжимающими. Из оценок $(24),(25)$ и (28) заключаем, что для операторов в (22) существует единственная пара неподвижных точек (см., напр. [25], стр. 389 - 401). Следовательно, СУ (22) имеет единственную пару решений $\{u(t) \in C(\Omega), \beta \in R\}$. Подставляя эти решения СУ (22) в (19), определим вторую величину восстановления $\varphi_{2}$. Кроме того, справедливы оценки скорости сходимости

$$
\begin{gathered}
\left|\beta^{j+1}-\beta\right| \leq \frac{\rho^{j+1}}{1-\rho}\left(\omega_{2}+\varepsilon_{2}\right) M_{0}, \\
\left\|u^{j+1}(t)-u(t)\right\| \leq \frac{\rho^{j+1}}{1-\rho}\left(\omega_{2}+\varepsilon_{2}\right) M_{0} .
\end{gathered}
$$

Нетрудно убедиться, что $u(t) \in C^{2}(\Omega)$. Теорема доказана.

Покажем, что множество интегро-дифференциальных уравнений (1), для которых выполняется последнее условие теоремы 1 , не пусто. Если в качестве примера берем функцию

$$
H(t)=e^{-w\left(\varepsilon_{2}+\omega_{2}\right) t},
$$

то это условие приобретает очевидный вид

$$
\rho=\left(1-e^{-w\left(\varepsilon_{2}+\omega_{2}\right) T}\right)<1 .
$$




\section{4. Устойчивость решения уравнения (1) по функциям восстановления}

Поскольку неизвестные величины определяются методом последовательных приближений, ставится вопрос об устойчивости решения уравнения (1) по величинам восстановления: $\beta$ и $\varphi_{2}$.

Теорема 2. Пусть выполняются условия теоремы 1. Если $u(t) \in C^{2}(\Omega) u \beta \in R$ - единственная пара решений $C У(22)$ и $\varphi_{2} \in R$ - вторая величина восстановления, то решение уравнения (1) $u(t)$ устойчиво по величинам восстановления: $\beta(x) u \varphi_{2}(x)$.

Доказательство. Пусть $u_{1}(t)$ и $u_{2}(t)$ - два различных решения краевой задачи $(1),(2)$, соответствующие двум различным значениям величин восстановления $\varphi_{21}$ и $\varphi_{22}, \beta_{1}$ и $\beta_{2}$, соответственно.

Положим, что

$$
\left|\varphi_{21}-\varphi_{22}\right|<\delta_{1},\left|\beta_{1}-\beta_{2}\right|<\delta_{2}, 0<\delta_{k}=\text { const, } k=1,2 \text {. }
$$

Тогда с учетом это, в силу условий теоремы, из (16) имеем

$$
\begin{gathered}
\left\|u_{1}(t)-u_{2}(t)\right\| \leq\left|\varphi_{21}-\varphi_{22}\right|\left\|Q_{2}(t)\right\|+\left|\beta_{1}-\beta_{2}\right|\left\|Q_{3}(t)\right\|+ \\
+\delta_{0}\left\|Q_{4}(t)\right\|\left(\left|\beta_{1}-\beta_{2}\right|+\left\|u_{1}(t)-u_{2}(t)\right\|\right)< \\
<\delta_{1}\left\|Q_{2}(t)\right\|+\delta_{2}\left[\left\|Q_{3}(t)\right\|+\delta_{0}\left\|Q_{4}(t)\right\|\right]+\delta_{0}\left\|Q_{4}(t)\right\|\left\|u_{1}(t)-u_{2}(t)\right\| .
\end{gathered}
$$

По условию теоремы $1-\delta_{0}\left\|Q_{4}(t)\right\|>0$. Поэтому из последнего неравенства получаем, что

$$
\left\|u_{1}(t)-u_{2}(t)\right\|<\frac{\delta_{1}\left\|Q_{2}(t)\right\|+\delta_{2}\left[\left\|Q_{3}(t)\right\|+\delta_{0}\left\|Q_{4}(t)\right\|\right]}{1-\delta_{0}\left\|Q_{4}(t)\right\|} .
$$

Отсюда окончательно получаем утверждения теоремы, если положим

$$
\varepsilon=\frac{\delta_{1}\left\|Q_{2}(t)\right\|+\delta_{2}\left[\left\|Q_{3}(t)\right\|+\delta_{0}\left\|Q_{4}(t)\right\|\right]}{1-\delta_{0}\left\|Q_{4}(t)\right\|}
$$

\section{Список литературы}

[1] Банг Н.Д., Чистяков В.Ф., Чистякова Е.В. О некоторых свойствах вырожденных систем линейных интегро-дифференциальных уравнений. I // Известия Иркутского государственного университета. Серия: Математика. 2015. Т. 11. C. $13-27$.

[2] Быков Я.В. О некоторых задачах теории интегро-дифференциальных уравнений. Фрунзе: Изд-во Кирг. гос. ун-та, 1957. 327 с. 
[3] Вайнберг М.М. Интегро-дифференциальные уравнения // Итоги науки. Математический анализ. Теория вероятностей. Регулирование. 1962. М.: ВИНИТИ, 1964. С. 5-37.

[4] Васильев В.В. К вопросу о решении задачи Коши для одного класса линейных интегро-дифференциальных уравнений // Известия высших учебных заведений. Математика. 1961. № 4. С. 8-24.

[5] Власов В.В., Перез Ортиз Р. Спектральный анализ интегродифференциальных уравнений, возникающих в теории вязкоупругости и теплофизике // Математические заметки. 2015. Т. 98, № 4. С. 630-634.

[6] Кривошеин Л.Е. Об одном методе решения некоторых линейных интегродифференциальных уравнений // Известия высших учебных заведений. Математика. 1960. № 3. С. 168-172.

[7] Ландо Ю.К. Краевая задача для линейных интегро-дифференциальных уравнений типа Вольтерра в случае распадающихся краевых условий // Известия высших учебных заведений. Математика. 1961. № 3. С. 56-65.

[8] Фалалеев М.В. Интегро-дифференциальные уравнения с фредгольмовым оператором при старшей производной в банаховых пространствах и их приложения // Известия Иркутского государственного университета. Серия: Математика. 2012. Т. 5, № 2. С. 90-102.

[9] Денисов А.М. Введение в теорию обратньг задач. М.: МГУ, 1994. 285 с.

[10] Костин А.Б. Обратная задача восстановления источника в параболическом уравнении по условию нелокального наблюдения // Математический сборник. 2013. T. 204, № 10. C. 3-46.

[11] Лаврентьев М.М., Савельев Л.Я. Линейные операторы и некорректные задачи. М.: Наука, 1991. 331 с.

[12] Прилепко А.И, Костин А.Б. О некоторых обратных задачах для параболических уравнений с финальным и интегральным наблюдением // Математический сборник. 1992. Т. 183, № 4. С. 49-88.

[13] Прилепко А.И., Тихонов И.В. Восстановление неоднородного слагаемого в абстрактном эволюционном уравнении // Известия Российской академии наук. Серия математическая. 1994. Т. 58, № 2. С. 167-188.

[14] Прилепко А.И., Ткаченко Д.С. Свойства решений параболического уравнения и единственность решения обратной задачи об источнике с интегральным переопределением // Журнал вычислительной математики и математической физики. 2003. Т. 43, № 4. С. 562-570.

[15] Романов В.Г. Обратные задачи для математической физики. М.: Наука, 1984. 264 c.

[16] Юлдашев Т.К. Обратная задача для одного нелинейного интегродифференциального уравнения третьего порядка // Вестник Самарского государственного университета. Естественнонаучная серия. 2013. № 1. С. 58-66. 
[17] Юлдашев Т.К. Об обратной задаче для нелинейных интегродифференциальных уравнений высшего порядка // Вестник Воронежского государственного университета. Серия: Физика. Математика. 2014. № 1. C. $153-163$.

[18] Юлдашев Т.К. Обратная задача для одного интегро-дифференциального уравнения Фредгольма в частных производных третьего порядка // Вестник Самарского государственного технического университета. Серия: Физикоматематические науки. 2014. Т. 34, № 1. С. 56-65.

[19] Гордезиани Д.Г., Авалишвили Г.А. Решения нелокальных задач для одномерных колебаний среды // Математическое моделирование. 2000. Т. 12, № 1. C. 94-103.

[20] Тихонов И.В. Теоремы единственности в линейных нелокальных задачах для абстрактных дифференциальных уравнений // Известия Российской академии наук. Серия математическая. 2003. Т. 67, № 2. С. 133-166.

[21] Пулькина Л.С. Нелокальная задача для гиперболического уравнения с интегральными условиями 1 рода с ядрами, зависящими от времени // Известия высших учебных заведений. Математика. 2012. № 10. С. 32-44.

[22] Юлдашев Т.К. Об одном интегро-дифференциальном уравнении Фредгольма в частных производных третьего порядка // Известия высших учебных заведений. Математика. 2015. № 9. С. 74-79.

[23] Yuldashev T.K. A double inverse problem for a partial Fredholm integrodifferential equation of fourth order // Proceedings of the Jangjeon Mathematical Society. 2015. Vol. 18, № 3. Pp. 417-426.

[24] Юлдашев Т.К. Обратная задача для нелинейных интегро-дифференциальных уравнений типа Benney-Luke с вырожденным ядром // Известия высших учебных заведений. Математика. 2016. № 9. С. 59-67.

[25] Треногин В.А. Функциональный анализ. М.: Наука, 1980. 495 с.

\section{Библиографическая ссылка}

Юлдашев T.K. Обратная задача для обыкновенного интегродифференциального уравнения с вырожденным ядром и нелокальными интегральными условиями // Вестник ТвГУ. Серия: Прикладная математика. 2016. № 3. С. 19-33.

\section{Сведения об авторах}

\section{1. Юлдашев Турсун Камалдинович}

доцент кафедры высшей математики Сибирского государственного аэрокосмического университета имени академика М.Ф. Решетнева.

Россия, 660014, г. Красноярск, проспект имени газеты «Краснолрский рабочиนั», 31, СибГАУ им. М. Ф. Решетнева. E-mail: tursun.k.yuldashev@gmail.com 


\title{
INVERSE PROBLEM FOR AN ORDINARY INTEGRO-DIFFERENTIAL EQUATION WITH DEGENERATE KERNEL AND NONLOCAL INTEGRAL CONDITIONS
}

\author{
Yuldashev Tursun Kamaldinovich \\ Associate professor at Higher Mathematics Department, \\ Reshetnev Siberian State Aerospace University \\ Russia, 660014, Krasnoyarsk, 31 Krasnoyarskiy rabochiy avenue, SibSAU. \\ E-mail: tursun.k.yuldashev@gmail.com
}

\begin{abstract}
Received 10.09.2016, revised 30.09.2016.
This article considers the questions of one value solvability of the nonlocal inverse problem for restore the resource and boundary regimes for a nonlinear Fredholm second-order ordinary integro-differential equation with degenerate kernel. By denoting the integro-differential equation is reduced to a system of algebraic equations with nonlinear right-hand side. This system is solved by the aid of modified Kramer method. By the aid of the given additional conditions is obtained the system of two equations with respect to first and second unknown quantity. Is derived also the formula for calculation the third unknown quantity. It is proved the one value solvability of this system by the method of successive approximations. Is derived also the formula for calculation the third unknown quantity. It is studied the stability of solution of the integro-differential equation with respect to restore quantities.
\end{abstract}

Keywords: nonlocal inverse problem, integro-differential equation, degenerate kernel, system of algebraic equations, one valued solvability.

\section{Bibliographic citation}

Yuldashev T.K. Inverse problem for an ordinary integro-differential equation with degenerate kernel and nonlocal integral conditions. Vestnik TvGU. Seriya: Prikladnaya Matematika [Herald of Tver State University. Series: Applied Mathematics], 2016, no. 3, pp. 19-33. (in Russian)

\section{References}

[1] Bang N.D., Chistyakov V.F., Chistyakova E.V. On some properties of degenerate system of linear integro-differential equations. I. Izvestija Irkutskogo gosudarstvennogo universiteta. Serija Matematika [Herald of Irkutsk State University. Series: Mathematics], 2015, vol. 11, pp. 13-27. (in Russian) 
[2] Bykov Ja.V. O nekotorykh zadachah teorii integro-differentsial'nykh uravnenij [On some problems of the theory of integro-differential equations]. Frunze, 1957. 327 p. (in Russian)

[3] Vajnberg M.M. Integro-differential equations. Itogi Nauki. 1962 [Results of Science], 1964, pp. 5-37. (in Russian)

[4] Vasil'jev V.V. To the problem of solution of the Cauchy problem for a class of linear integro-differential equations. Izvestija Vuzov. Matematika [Russian Mathematics], 1961, no. 4, pp. 8-24. (in Russian)

[5] Vlasov V.V., Perez Ortiz R. Spectral analysis of integro-differential equations in viscoelasticity and thermal physics. Mathematical Notes, 2015, vol. 98 (3), pp. 689-693.

[6] Krivoshein L.E. On a method of solving the some integro-differential equations. Izvestija Vuzov. Matematika [Russian Mathematics], 1960, no. 3, pp. 168-172. (in Russian)

[7] Lando Yu.K. Boundary value problem for Volterra linear integro-differential equations in the case of disjoint boundary value conditions. Izvestija Vuzov. Matematika [Russian Mathematics], 1961, no. 3, pp. 56-65. (in Russian)

[8] Phalaleev M.V. Integro-differential equations with Fredholm operator on the highest derivative in Banach spaces and their applications. Izvestija Irkutskogo gosudarstvennogo universiteta. Serija Matematika [Herald of Irkutsk State University. Series: Mathematics], 2012, vol. 5 (2), pp. 90-102. (in Russian)

[9] Denisov A.M. Vvedenie v Teoriju Obratnyh Zadach [Introduction to the Theory of Inverse Problem]. Lomonosov Moscow State University, Moscow, 1994. 285 p.

[10] Kostin A.B. The inverse problem of recovering the source in a parabolic equation under a condition of nonlocal observation, Sbornik: Mathematics, 2013, vol. 204 (10), pp. 1391-1434.

[11] Lavrent'ev M.M., Savel'ev L.Ya. Lineynye operatory i nekorrektnye zadachi [Linear operators and ill-posed problems]. Nauka, Moscow, 1991. 331 p.

[12] Prilepko A.I., Kostin A.B. On certain inverse problems for parabolic equations with final and integral observation. Sbornik: Mathematics, 1992, vol. 75 (2), pp. 473-490.

[13] Prilepko A.I., Tikhonov I.V. Restoring nonhomogeneous term in an abstract evolution equation. Izv. RAN. Seriya matematicheskaja [Russian Academy of Sciences. Izvestiya Mathematics], 1994, vol. 58 (2), pp. 167-188. (in Russian)

[14] Prilepko A.I., Tkachenko D.S. Properties of solutions of a parabolic equation and the uniqueness of the solution of the inverse source problem with integral over determination. Computational Mathematics and Mathematical Physics, 2003, vol. 43 (4), pp. 537-546. 
[15] Romanov V.G. Obratnye Zadachi Matematicheskoj Fiziki [Inverse Problems of Mathematical Physics]. Nauka, Moscow, 1984. 264 p.

[16] Yuldashev T.K. Inverse problem for a nonlinear integro-differential equation of the third order. Vestnik Samarskogo gosudarstvennogo universiteta. Seriya: Estestvennonauchnaya [Herald of Samara state university. Series: Natural Sciences], 2013, no. 1, pp. 58-66. (in Russian)

[17] Yuldashev T.K. An inverse problem for nonlinear integro-differential equations of higher order. Vestnik Voronezhskogo gosudarstvennogo universiteta. Seriya: Fizika. Matematika [Herald of Voronezh state university. Physics. Mathematics], 2014, no. 1, pp. 153-163. (in Russian)

[18] Yuldashev T.K. Inverse problem for a partial Fredholm integro-differential equation of the third order. Vestnik Samarskogo gosudarstvennogo tekhnicheskogo universiteta. Serija: Fiziko-matematicheskie nauki [Herald of Samara State Technical University. Series: Physics \& Mathematics Sciences], 2014, vol. 34 (1), pp. 56-65. (in Russian)

[19] Gordeziani D.G., Avilishbili G.A. Solving the nonlocal problems for onedimensional medium oscillation. Matematicheskoe Modelirovanie [Mathematical Modelling], 2000, vol. 12 (1), pp. 94-103. (in Russian)

[20] Tikhonov I.V. Uniqueness theorems for linear nonlocal problems for abstract differential equations. Izvestiya: Mathematics, 2003, vol. 67 (2), pp. 333-363.

[21] Pul'kina L.S. A nonlocal problem for a hyperbolic equation with integral conditions of the 1st kind with time-dependent kernels. Russian Mathematics, 2012, vol. 56 (10), pp. 26-37.

[22] Yuldashev T.K. A certain Fredholm partial integro-differential equation of the third order. Russian Mathematics, 2015, vol. 59 (9), pp. 62-66.

[23] Yuldashev T.K. A double inverse problem for a partial Fredholm integrodifferential equation of fourth order. Proceedings of the Jangjeon Mathematical Society, 2015, vol. 18 (3), pp. 417-426.

[24] Yuldashev T.K. Inverse problem for a nonlinear Benney-Luke type integrodifferential equations with degenerate kernel. Russian Mathematics, 2016, vol. 60 (9), pp. 53-60.

[25] Trenogin V.A. Funktsional'nyj Analiz [Function Analysis]. Nauka, Moscov, 1980. 495 p. (in Russian) 